\title{
TENDANCES ÉVOLUTIVES DU LATIN TARDIF DANS LA BRITANNIA ROMAINE (D'APRÈS LES INSCRIPTIONS)
}

\begin{abstract}
Summary: This study presents some of the most important phonological and grammatical phenomena which show the evolution of Late Latin in the Roman province of Britannia. The investigation is based on a corpus of inscriptions on stone (established by Collingwood and Wright). The Vulgar Latin of Britannia seems not very different from that of other provinces, but the progression of certain changes is slower. The author insists on the different origins of soldiers and colonists who took part in the romanization of the island.
\end{abstract}

Key words: Britannia, Late Latin, Epigraphy, Phonology, Morphosyntax

On trouvera ici la liste des phénomènes linguistiques les plus importants qui représentent, sur les inscriptions latines provenant de la province romaine de Britannia, des écarts par rapport à ce qu'il est convenu d'appeler l'usage classique du latin. Les données épigraphiques proviennent du recueil publié par Collingwood et Wright en $1965^{1}$ et contenant un peu plus de deux mille inscriptions. La question que je me pose est la question que se sont posée, à propos de la latinité des différentes provinces, tous ceux qui s'intéressaient à la différenciation régionale de la langue ${ }^{2}$ : est-il possible de relever, dans le corpus donné, des particularités susceptibles de modifier l'image d'ensemble que nous nous formons de l'évolution du latin des siècles postclassiques ?

Par définition, les matériaux linguistiques fournis par les inscriptions sont limités; ils laissent apercevoir avant tout des phénomènes phonétiques et morphologiques, avec certains faits lexicaux ; à cause du caractère souvent schématique de la formulation, il est plus difficile de découvrir d'éventuelles modifications diachroniques dans la construction de la phrase et du texte. L'onomastique nécessite naturelle-

\footnotetext{
${ }^{1}$ Collingwood, R. G. - Wright, R. P. : The Roman Inscriptions of Britain. Oxford 1965. Sauf indication contraire, les numéros d'inscriptions renvoient à cette édition.

${ }^{2}$ Cf. notamment HeRmAn, J. : Aspects de la différenciation territoriale du latin sous l'Empire (1965). In HERMAN, J. : Du latin aux langues romanes. Tübingen 1990, 10-28.
} 
ment un traitement séparé : elle nous renseigne avant tout sur les usages sociaux et de façon secondaire seulement sur les changements linguistiques; néanmoins, les anthroponymes d'origine étrangère, quand ils apparaissent sur les inscriptions, peuvent être différemment adaptés au système latin, et ils deviennent alors intéressants du point de vue de la langue latine elle-même. Ici vont suivre quelques observations de nature phonétique, complétées par un petit nombre de remarques grammaticales.

Pour commencer par un phénomène bien connu du vocalisme latin tardif, les voyelles $\bar{e}$ et $\breve{l}$ de la période classique tendent à coïncider en un $e$ fermé (les continuations romanes de tèla, fidem $>$ it. tela, fede, fr. toile, foi témoignent clairement de l'aboutissement de cette évolution). Les inscriptions latines peuvent refléter cette nouvelle prononciation en confondant les lettres $E$ et $I$, ce qui constitue une " faute » caractéristique dans la graphie des mots contenant un $\bar{e}$ ou un $\breve{l}$ classiques. Dans nos matériaux, le phénomène apparaît surtout en syllabe inaccentuée. Les données sûres se répartissent en trois groupes : 1. Le substantif ciuis est souvent noté CIVES : 159 CIVES HISP(anus) CAVRIE(n)SIS [= de Caurum, Lusitanie] ; 621 CIVES DOBVN$N A$ [= de la tribu insulaire des Dobunni] ; sur 108, on lit CIVES RAVR(icus) [cf. Augusta Raurica, en Germanie Supérieure], peut-être à côté d'un IT (pour et). Sur ces mêmes inscriptions, on trouve des $E$ et des $I$ marqués correctement. 2. Nous relevons $E$ pour $I$ dans la partie prétonique de deux mots polysyllabiques : 712 AVREFICI$N A M$ et 221 SIGNEFICATVR (ce dernier sur une defixio). 3. La tendance peut être relevée sporadiquement dans des noms propres : 1586 SVPERSTIS pour Superstes ; 509 MACID (abréviation pour leg. Macedonicae); 628 AVR(elius) SEN[o]PIANVS [de Sinope, ville d'Asie Mineure]. Dans certains cas, la confusion semble s'étendre à la voyelle $\bar{l}$ longue : 143 EVTVCHES LEB (= lïbertus); 306 DEMEDIAM PARTEM (= dimidiam) ; dans ce dernier cas, il s'agit d'une defixio présentant des fautes diverses, parmi lesquelles PERDEDIT pour perdidit est éventuellement une forme recomposée. Laissons de côté pour le moment les flottements d'ordre grammatical, telle l'alternance $-e / i$ dans les terminaisons du datif et de l'ablatif : 307 DEO NVDENTE (= Nudenti) ; 1981 INSTA(nte) (...) SEVERO PRINCIPI (= principe).

Les voyelles $\bar{e}$ et $\bar{l}$ peuvent naturellement coïncider en syllabe accentuée aussiquoique le nombre des données sûres ne soit pas très élevé. Pour une prononciation plus ouverte de $\breve{l}$, rappelons 306 DEMEDIAM (déjà cité) ; ajoutons 978 BASELICAM (inscription votive pour l'empereur Sévère Alexandre, de l'an 222, avec un texte par ailleurs correct). Le cas de 154 [al]LE ATUM est plus problématique : c'est une defixio, avec les mots notés à l'envers, les éditeurs lisent donc muta el[la] (pour illa) et traduisent 'elle doit devenir muette'. Curieusement, un certain nombre de $\breve{e}$ accentués sont notés $I: 631$ MINTLA (= mentula); 2311 SIMPER (= semper), avec peut-être 605 LIPIDO (= Lepido, mais sujet à caution à cause d'une fracture de la pierre) et 1921 SIPTIMO (= Septimo, connu seulement par tradition manuscrite). Un $\bar{l}$ accentué semble être rendu par la lettre $E$ dans 306 DEVO NODENTI, nous ne devons cependant pas exclure ici une interférence lexicale entre deo et dīuo. Pourtant, cette tablette d'exécration offre, comme on l'a vu, plusieurs graphies témoignant de la confusion $e \sim i$ (DEMEDIAM pour dimidiam et peut-être PERDEDIT pour perdidit). Cette sorte d'instabilité graphique - et probablement une instabilité phonétique 
qu'elle reflète - est clairement illustrée par le flottement VETERIS VITIRIS (nom d'une divinité celtique) ; rappelons, à titre d'exemple, 1139 DEO VETERI $\sim 1140$ DEO VITIRI.

Constatons donc que, dans l'ensemble, la tendance à la confusion entre les voyelles classiques $\bar{e}$ et $\breve{l}$ est bien présente dans la province de Britannia, mais ses progrès semblent plutôt lents, par rapport à ce que l'on constate dans d'autres régions de l'Empire. On peut en dire de même concernant la tendance parallèle que le latin tardif manifeste dans le domaine des voyelles vélaires - il s'agit, bien entendu, de la coïncidence des voyelles classiques $\bar{o}$ et $\breve{u}$ en un $o$ fermé (rappelons les continuations romanes de flörem, gŭla $>$ it. fiore, gola, fr. fleur, gueule). Rares sont les exemples dans notre province qui soient absolument dignes de foi ; encore ceux-ci se trouventils dans des noms propres (peut-être moins liés à une image graphique familière). Ainsi, la lettre $O$ note la voyelle $\breve{u}$ deux fois dans le nom de l'unité militaire ala Astürum (de Astüres) : 266 [A]STOR(um); 1337 ASTO(rum); et une fois nous trouvons $V$ pour $\bar{o}$ dans le nom de l'ala Sebosiana : 605 ALAE SEBVSSIAN $(a e)^{3}$. Les autres données dont nous disposons sont moins convaincantes. Le nom de la divinité Nodens (ou Nodons) se trouve écrit une fois avec V: 307 DEO NVDENTE (= deo Nudenti); au lieu de Vibuleius, forme habituelle, ${ }^{4}$ nous avons le nom avec $O: 1052$ P. VIBOLEIVS SECVNDVS; pour le nom du tribunus qui s'appelle en latin Flauius Titianus, la pierre a conservé une transcription grecque, qui remplace les terminai-

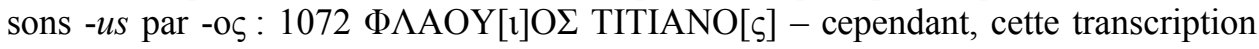
peut avoir un caractère "morphologique». Le petit nombre des exemples semble montrer que la différence, à l'origine phonologique, entre les voyelles $\bar{o}$ et $\breve{u}$ commence à se déphonologiser dans la province de Britannia également, mais il ne s'agit, en fait, que d'un commencement.

En ce qui concerne les consonnes, nous devons naturellement tenir compte du fait que leur évolution dépend, dans une grande mesure, de leur place dans la syllabe et dans le mot dont elles font partie. Nous nous intéresserons donc surtout aux positions dites «faibles », où les consonnes sont exposées à des altérations caractéristiques. D'après nos connaissances du phonétisme du latin tardif, ce sont notamment les consonnes finales de mot qui se trouvent dans une telle position «menacée ». Il est très probable que dans l'usage non littéraire de la période postclassique, $-m$ final n'avait plus aucune existence phonétique (certains monosyllabes exceptés) : ainsi, son absence fréquente sur les inscriptions de Britannia indique une extension considérable du latin vulgaire dans la province et une romanisation linguistique relativement profonde des territoires romains de l'île. Soit dit en passant que malgré le rôle morphologique évident dont $-m$ final se trouve chargé en latin classique, sa disparition n'entraîne pas, dans la plupart des cas, de "catastrophe communicative ", il s'agit donc d'un élément au degré de redondance élevé. Citons 1016 ARA FECIT; 1776 DO (= deo) BLATVCADRO VOTV S(olutum); 1912 IN LABE CONL(apsum)

\footnotetext{
${ }^{3}$ Pour un cognomen Sebosus (qui pourrait signifier à l'origine 'graisseux'), cf. SCHULZE, W. : Zur Geschichte lateinischer Eigennamen. Berlin 1904, 277.

${ }^{4}$ Cf. le commentaire des éditeurs et SCHulze (n. 3) 380.
} 
[sur une inscription officielle]; 293 ANORV LII ; 326 CVAMPEIANO (= cum Ampeiano). On peut ajouter le type d'ablatif absolu formulaire représenté par $360 C V R A$ AGENTE AMANDA CONIUGE (au lieu de curam, cf. également 365); la formule, dont la structure grammaticale s'était déjà peut-être obscurcie pour les locuteurs, pouvait perdre son - $m$ de l'accusatif, tout en gardant sa valeur sémantique.

Nous sommes, comme toujours, embarrassés en abordant la situation de $-s$ final. Il a toujours été tentant de rechercher, dans les matériaux épigraphiques latins, des traits qui préfigurent la future répartition géographique des « traitements » de $-s$ final (conservation dans l'Ouest et suppression dans l'Est de la Romania, cf. tres $>$ esp. tres, it. tre). Force est de constater cependant que les inscriptions latines ne laissent pas prévoir une telle dialectalisation et que, pour diverses raisons, il est difficile de juger des progrès de la chute de $-s$ final à la période du latin tardif. ${ }^{5}$ De toutes manières, les données que nous offrent les inscriptions de notre province sont elles-mêmes incertaines. Il est banal de constater d'abord qu'un $-s$ peut manquer à la fin d'une ligne du texte épigraphique, du fait d'une abréviation ou tout simplement par faute de place (par ailleurs, sur les inscriptions que nous citerons ici, $-s$ final peut très bien se maintenir dans d'autres mots, à d'autres endroits du texte) : 600 IVLIV| IANVARIVS ; 993 AELIV | VITALIANVS ; 1003 IANVARIVS LVCANV (fin de l'inscription). Une interférence grammaticale (confusion du génitif et du datif) n'est pas à exclure dans 2019 TREBONI NEPOTI (il s'agit probablement de la centuria de Trebonius Nepos). Pour 9 NA(tus) ATHENI (= Athenis ?), la leçon elle-même est malheureusement incertaine. On ne relève donc, pour la province, aucune tendance à l'élimination des $-s$ : à cet égard, le langage de la Britannia est décidément conservateur.

Les consonnes dites « implosives »- consonnes intérieures en fin de syllabe, c'est-à-dire premiers éléments d'un groupe consonantique - occupent également une position «faible", dans le tableau d'ensemble des évolutions latines postclassiques. En d'autres termes, la portion postvocalique de la syllabe acquiert souvent une réalisation plus ouverte ou peut être encline à disparaître. Ainsi, le groupe $n s$ s'est partout simplifié dans l'Empire et devait se prononcer $s$ dès l'époque classique peutêtre, dans le langage non littéraire ; avec les graphies comme MENSIS, la norme orthographique pouvait naturellement se maintenir. Dans nos matériaux, $-n$ tombe parfois devant d'autres consonnes aussi : 924 SACTO (= sancto); 7 METEM (= mentem); et dans le nom d'une ciues Dobunna: 621 VERECVD.RVFILIA (= Verecun$d a$ ). La consonne $m$ a un comportement pareil dans 686 SEPRONIE, SEPRONIVS (pour Semproniae, Sempronius). La simplification des groupes de trois consonnes, phénomène bien connu partout dans l'Empire, s'inscrit dans la même tendance fondamentale : 151 SCVLTOR (= sculptor), 369 DEFVNTVS (= defunctus); peut-être également 1334 CAMPESR(i)B(us) (qui représente Campestribus, mais sous la forme d'une abréviation, en fin de ligne).

Pour des raisons rythmiques, nous pouvons admettre une sorte de parallélisme entre le comportement des groupes consonantiques et celui des consonnes longues.

\footnotetext{
${ }^{5}$ Cf. HeRman, J. : La disparition de $-s$ et la morphologie dialectale du latin parlé (1987). In HeRMAN, J. : Du latin aux langues romanes II. Tübingen 2006, 33-42. 
En effet, la simplification des groupes de deux consonnes et la dégémination conduisent au même résultat, notamment à la suppression de la « coda » syllabique. On sait naturellement jusqu'à quel point la transcription des consonnes longues est hésitante sur les inscriptions - il est toutefois possible d'attribuer une valeur phonétique à certaines graphies dégéminées offertes par nos matériaux de Britannia. D'abord, les données du type $A N I S, A N O S, A N O R(u m)$ sont très fréquentes ; citons en outre 156 IVLIVS VITALIS ... ANOR(um) XXIX ... EX COLEGIO FABRICE(nsium); 959 et 1131 APOLINARIS (notons que les mots annorum, collegio et Apollinaris ont subi ici le changement défini par le soi-disant « mamilla-Gesetz $»^{6}$ ). La chute de $-m$ final, ainsi que la simplification de certains groupes consonantiques et de certaines géminées montrent qu'une tendance favorisant les syllabes ouvertes aux dépens des syllabes fermées - tendance postclassique bien connue par ailleurs - prend son essor dans la province de Britannia, durant les quelques siècles de domination romaine.

On comprend fort bien que la dégémination soit souvent mise en rapport avec le soi-disant « affaiblissement intervocalique » qui caractérise certains dialectes préromans et une partie des langues romanes - même si la nature exacte de ce rapport n'est pas parfaitement claire. De toutes manières, l'assimilation intervocalique en question - qui transformerait notamment $p, t, k$ en $b, d, g$ dans cette position - ne caractérise pas nos matériaux de Britannia; le flottement que peut présenter l'usage des lettres $C$ et $G$ doit être d'origine purement graphique et sans valeur phonétique. Toutefois, nous disposons d'un petit nombre de données représentant un phénomène bien connu du latin tardif, qui peut être mis en rapport avec les affaiblissements intervocaliques plus tardifs : il s'agit de la confusion phonologique de deux phonèmes distincts en latin classique, l'occlusive bilabiale sonore $/ b /$ et la fricative labiovélaire sonore $/ w /$. Comme on pouvait s'y attendre d'après les témoignages épigraphiques des autres provinces, le résultat de cette confusion est marqué à l'intervocalique par la lettre $V: 17 D$ (is) M(anibus) VIVIO (Vibio). En contrepartie, pour ainsi dire - et toujours en accord avec une tendance existant dans le préroman -, c'est la lettre $B$ qui représente l'ancien phonème $/ w /$ à l'initiale du mot dans 1 HOMINIBVS BAGIS BITAM (= uagis uitam, sur une inscription qui semble être dédiée à Mithra ${ }^{7}$ ).

Il existe certainement un domaine de l'évolution linguistique où les inscriptions de Britannia n'accusent pour ainsi dire aucun retard par rapport aux autres provinces de l'Empire. En effet, certains changements phonétiques, caractéristiques de la période du latin tardif et modifiant la structure syllabique du mot, peuvent être attribués à une vitesse accrue de la parole. Ce tempo plus rapide agit directement sur les voyelles pénultièmes atones et sur les voyelles en hiatus, qu'il tend à éliminer. En ce qui concerne d'abord ces dernières, la graphie $I$ pour un $e$ prévocalique est un indice de la consonantification de la voyelle en hiatus. Ainsi, dans 1320 OCIANO (= Oceano), 1303 DIO (= deo), 1525 et 1543 DIE (= deae), nous avons certainement la séquence phonétique « $j+$ voyelle $»$. Or, la fricative $j$ qui se développe dans cette

\footnotetext{
${ }^{6}$ LeumanN, M. : Lateinische Laut- und Formenlehre. München 1977, 184 (examen critique du problème). Cf. NiedermanN, M. : Précis de phonétique historique du latin. Paris $1953^{4}, 116$.

${ }^{7}$ Les éditeurs interprètent '(give) life to men who wander'. Cf. Vermaseren, M. J. : Corpus inscriptionum et monumentorum religionis Mithriacae. The Hague 1956, 287.
} 
position pourra, par la suite, disparaître ou se combiner avec la consonne qui la précède. Nous pouvons certes avoir le résultat d'une contraction « $e+e$ » dans 1449, 1528, $1537 D A E$ (pour deae), cependant 1776 et $1784 D O$ (pour deo, sur deux tablettes votives dédiées à une divinité celtique), $2276 P O$ (= pio), 1175 DONISIVS (= Dionisius) et peut-être 1696 PROVINCIAE SVPERORS (= Superioris) témoignent la chute de la consonne $j$, tandis que dans 764 CALEZIVS (pour le nom connu par ailleurs sous la forme Caledius), la graphie $Z$ peut très bien représenter la combinaison consonantique $[d z]$, bien connue à partir de la période postclassique et résultant de la contraction de $j$ avec un $d$ précédent. L'autre fricative très ouverte du latin classique, le $[w]$ labiovélaire, peut disparaître à l'époque tardive même entre deux voyelles; le phénomène apparaît dans nos matériaux avec 2107 VELLAVS (= Vellauus, peut-être une haplographie) et 2238 PIAONIO (= Piauonio, dans le nom de l'empereur du III siècle, M. Piavonius Victorinus). Pour la syncope de la voyelle pénultième atone, citons 323 DOMNA ; 1570 PROCLI (= Proculi) ; ajoutons ici un cas de syncope prétonique : 478 VETRANO. Ce qui est plus surprenant (mais pas du tout inconnu dans d'autres provinces non plus), c'est le fait que la syncope peut conduire à la formation de groupes de consonnes inhabituels : 240 et 1396 DECMUS ; 631 MINTLA (= mentula). Il est difficile de décider si dans les diverses variantes du nom de la divinité celtique Belatucadrus / Balatucadrus / Blatucadrus, nous avons affaire à des syncopes ou à des épenthèses ; il peut très bien s'agir de plusieurs emprunts dispersés dans l'espace et échelonnés dans le temps.

Dans le domaine de la morphologie, nos matériaux sont beaucoup plus pauvres ; néanmoins, il n'est pas difficile d'y reconnaître la manifestation de certaines tendances bien connues de l'évolution grammaticale postclassique. On comprend bien le changement analogique représenté par 594 SOCAERE, pour socerae, datif d'un socera de la $\mathrm{I}^{\text {re }}$ déclinaison qui remplace socrus de la $\mathrm{IV}^{\mathrm{e}}$. Autre confusion analogique entre types de déclinaison : dibus pour $d \bar{i} s$ est fréquent (d'ailleurs en parallélisme évident avec le classique deabus) : 926 OMNIBVS DIBVS ; en liaison avec le nom de divinité Veteris / Vitiris : 1456, 1730, 1803 DIBVS VETERIBVS. Remarquons ici que l'emploi des cas peut subir une simplification, notamment lorsque, dans une coordination, le deuxième membre ne respecte pas la règle de l'accord, et une forme particulière de la déclinaison est économisée : 140 LOVCETIO MARTI ET NEMETONA (pour Nemetonae, « datif + nominatif » remplaçant « datif + datif »). En ce qui concerne la morphologie du verbe, nous relevons dans nos matériaux de nouvelles preuves pour le polymorphisme du parfait de ponere : 621,689 POSIT ; 1529 POSIVIT ; 1592 POSSIVIT ; 1606 [p]OSVVIT. ${ }^{8}$ Pour terminer cet aperçu grammatical nécessairement bref, signalons encore une forme de l' « économie syntaxique », notamment l'absence du démonstratif antécédent devant un pronom relatif : 306 INTER QUIBVS NOMEN SENICIANI, pour inter eos quibus (tablette d'exécration ; traduction des éditeurs : 'among those who are called Senicianus').

\footnotetext{
${ }^{8}$ Cf. Herman, J. : Posit (= Posuit) et questions connexes dans les inscriptions pannoniennes. Essai de géographie linguistique (1961). In HERMAN : Du latin aux langues romanes (n. 2) 94-104.
} 
$\mathrm{Au}$ cours de cette présentation du phonétisme et de la grammaire qui se dégagent des inscriptions de la province, nous avons déjà amorcé nos conclusions : la latinité de la Britannia connaît la plupart des tendances évolutives qui caractérisent la période du latin tardif, mais dans certains domaines, elle montre des hésitations et du retard ; elle est plutôt conservatrice. Deux faits d'arrière-plan doivent être rappelés en rapport avec ces constatations linguistiques : la soudaine rupture de la présence romaine dans l'île, au début $\mathrm{du} \mathrm{V}^{\mathrm{e}}$ siècle, donc la « continuation » à jamais abolie; et l'origine ethnique et géographique très mélangée des soldats et des colons qui devaient peupler et romaniser la province. L'effet «melting-pot» résultant de cette diversité devra faire un jour l'objet d'une investigation sociologique et sociolinguistique profonde.'

Sándor Kiss

Université du Debrecen

Département de Langue Française

H-4010 Debrecen

Egyetem tér 1.

Hongrie

${ }^{9}$ Cette étude a bénéficié de l'appui du projet OTKA no. K 62032 (Computerized Historical Linguistic Database of Latin Inscriptions of the Imperial Age). 